\title{
BINARY MORPHOLOGICAL SUBBAND DECOMPOSITION FOR IMAGE CODING
}

\author{
Metin Nafi Gürcan, Ömer N. Gerek, A. Enis Cetin \\ Bilkent University, Dept. of Electrical and Electronics Engineering, \\ Bilkent, Ankara TR-06533, Turkey \\ E-mail: gurcan@ee.bilkent.edu.tr \\ Phone: (90) 312-266 4307 Fax: (90) 312-266 4126
}

\begin{abstract}
In this paper a binary waveform coding method based on morphological subband decomposition coupled with embedded zero-tree and entropy coding is described. This method can be utilized in text compression or bit-plane coding of images. Binary morphological subband decomposition operations are carried out in the Gallois Field(2), resulting in a computationally efficient structure. Simulation studies are presented.
\end{abstract}

\section{INTRODUCTION}

The subband decomposition is frequently used in coding and pattern recognition applications. Traditionally, linear filters with perfect reconstruction (PR) property are employed for the decomposition. A nonlinear subband decomposition method for lossless image coding based on embedded zerotree structure is described in [1]. The key idea is to replace the linear filters in a classical subband decomposition scheme with nonlinear filters. If the input image is binary then such a decomposition can yield non-binary images, which may lead to higher compression ratios. In this paper, a nonlinear binary waveform coding method is developed. In this method, arithmetic operations are carried out in Gallois Field-2(GF(2)), hence the 'subband' images will be binary. This Nonlinear Binary Subband Decomposition (NBSD) can be used for cod-

This work is supported by TUBITAK (Turkish Scientific and Technical Research Council) Grant No. COST 249, and NSF Grant No. INT-9406954.

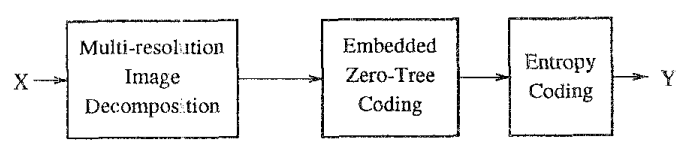

Figure 1: Binary Image Compression Steps

ing of binary images such as two-level texts, bit planes of images, etc.

The block diagram of the compression scheme is shown in Fig. 1. The first step is a binary multiresolution image decomposition which can be obtained either by using the NBSD or the binary wavelet transform (BWT) [2] of Swanson and Tewfik. Both the NBSD and the recently developed BWT have the perfect reconstruction property. The GF(2) arithmetic based NBSD structure is described in Section 2. After the binary subband decomposition, binary subband images at different resolution levels are produced. A zero-tree representation is used to take advantage of the self similar structure of the multiresolution subimages. This representation, first proposed by Shapiro [3], is similar to putting end-of-block marks in block based transform coding techniques. In our case the decomposed images are binary, therefore a modified form of zero-tree representation is used. The output of this representation has tree symbols $(0$, $1,2)$. These symbols are then binary encoded using Huffman encoding and the resulting bit-stream is packed into bytes. The coding scheme is explained in Section 3. Simulation results are presented in Section 4. 


\section{BINARY NONLINEAR SUBBAND DECOMPOSITION}

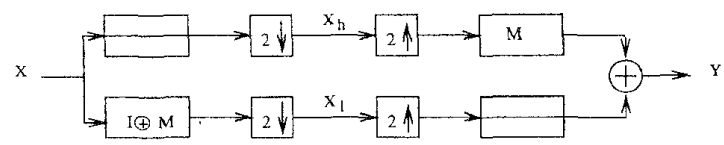

Figure 2: Nonlinear Binary Subband Decomposition

Figure 2 illustrates the nonlinear binary subband decomposition structure. In this scheme, all arithmetical operations are performed in $\mathrm{GF}(2)$ which guarantees that the resultant decomposed subimages are still binary. An important feature of this structure is that a nonlinear 'half-band' filter $M(x)$ is used instead of linear filters of traditional subband decomposition structures [1].

Let us review the concept of nonlinear halfband filter. The nonlinear filter $M$ is called a nonlinear half-band filter, if every other sample of the filter output $z$ is equal to a multiple of the input signal $x$, i.e.,

$$
z(2 k)=c x(2 k)
$$

where $\mathrm{k}$ is an integer, and $\mathrm{c}$ is an arbitrary constant. For example, a median filter with the region of non-compact support shown in Figure 3 can be used as a nonlinear half-band filter as follows:

$$
\begin{aligned}
z(2 k)= & c x(2 k) \\
z(2 k+1)= & \text { median }\{x(2 k-2), x(2 k), \\
& x(2 k+2), x(2 k+4)\}
\end{aligned}
$$

For instance, $z(3)$ is obtained from $z(0), z(2), z(4)$ and $z(6)$.

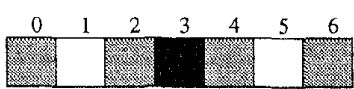

REGION OF SUPPORT

CURRENT PIXEL

Figure 3: Region of Support for 1-D Nonlinear Half-band Filter

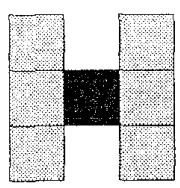

REGION OF SUPPORT

CURRENT PLXEL

Figure 4: Region of Support for 2-D Nonlinear Half-band Filter

In the decomposition part of the filter bank, the upper branch does nothing but downsamples the input signal by a factor of two. In the lower branch the input signal $x$ is filtered by $I \oplus M(x)$ where $I$ is the identity operator and $M(x)$ is a nonlinear half-band filter.

In the reconstruction part, the subsignal $x_{l}$, is first upsampled by a factor of two. The upsampled signal is filtered by the nonlinear filter $M(x)$. The other subsignal, $x_{h}$, is just upsampled by a factor of two. Finally, the resultant signals in the upper and lower branches are added together to give the reconstructed signal $y$. With the appropriate choice of the nonlinear half-band filter $M(x)$ and its region of support the reconstructed signal $y$ is the exact replica of the original signal $x$. This statement can be proved following the lines of proof in [5] and noting that none of the operations are field dependent. Hence, the structure in Figure 2 provides perfect reconstruction in GF(2) arithmetic, too.

The proposed decomposition structure can easily be extended to two dimensions by first performing the decomposition on the rows and then on the resultant columns. An example 2-D nonlinear half-band filter is the median filter with the region of support shown in Figure 4.

The BWT is another binary subband decomposition method. Swanson and Tewfik used this transformation for the subband decomposition of binary images [2]. The subband images in this decomposition resemble the binary images obtained by first taking the ordinary wavelet transform of the original binary image and then thresholding the resultant sub-images. The decomposition structure based on the BWT also employs GF(2) arith- 


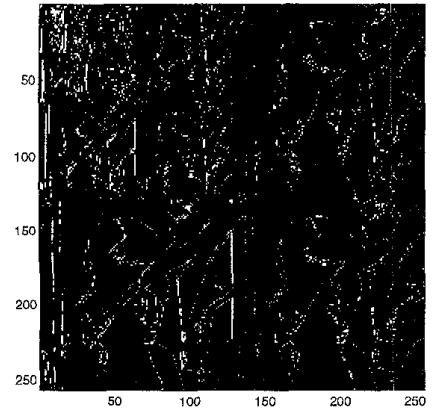

Figure 5: Binary Subband Decomposition of the most significant bit-plane of the Lena(256×256) image obtained by the Morphological Subband Decomposition

metic. Figures 5 and 6 show the binary decomposition of the most-significant bit plane of Lena (256x256) image, which are produced by the NBSD and the BWT, respectively.

\section{LOSSLESS CODING OF DECOMPOSED IMAGES}

The subband decomposition produces sub-band images with a sparse structure as shown in Figures 5 and 6 . We propose a modified form of embedded zero-tree coding to compress the decomposed images with many zero-valued pixels.

In this method, first the subband images are scanned in a zig-zag order, i.e the subbands lowhigh, high-low, and high-high $\left(L H_{l}, H L_{l}, H H_{l}\right)$ are examined at the $l$-th resolution level. If a pixel with zero value is encountered at location $(i, j)$ in one of the subbands, say $X X_{l}$ then all the pixel values at locations $\left(2^{k}(i-1)\right.$ to $2^{k} i, 2^{k}(j-1)$ to $\left.2^{k} j\right)$ in subbands $X X_{k}(k=1, \ldots, l-1)$ are checked for zeros. When all these pixels have zero values, the pixel at $(i, j)$ is called the root of a zero-tree, denoted by a symbol ' 2 ' (or any other value different from 0 or 1). An example zero-tree in a subband decomposed binary image is shown in Figure 7. Finally, the zero-valued pixels which are not roots of zero-trees are represented by '0's and all pixels with value 1 are represented by the symbol ' 1 '.

The zero-embedded coding produces a symbol stream with 0's, 1's and 2's. For a number of

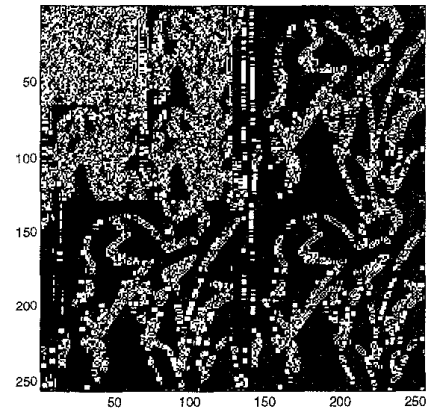

Figure 6: Binary Subband Decomposition of the most significant bit-plane of the Lena(256x256) image obtained by the Binary Wavelet Transform

sample images, it is observed that the number of 1 's is the smallest and the number of 2's is the largest. The coding theory suggests that the symbols ' 2 ' and ' 0 ' should be represented by two bits and the symbol ' 1 ' should be represented by one bit. Higher compression ratios can be achieved if the resultant bit stream are packed into bytes which are then run-length encoded.

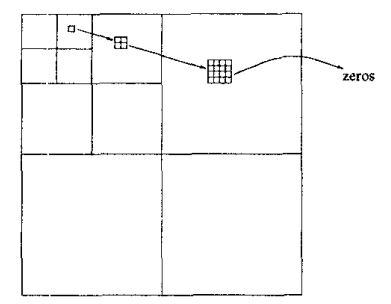

Figure 7: A sample zero-tree

\section{SIMULATION STUDIES}

The lossless compression scheme was applied to the compression of binary images. The first such image was the $256 \times 256$ pixels binary image of the letter "a." Figures 8 and 9 show the outputs of the NBSD and BWT applied this image for three times. The NBSD decomposed image was compressed 10.8 times with the lossless compression scheme. This result is better than a straightforward Lempel-Ziv (LZ) coding which yields a compression ratio of 7.19.

The second binary image was the most signif- 


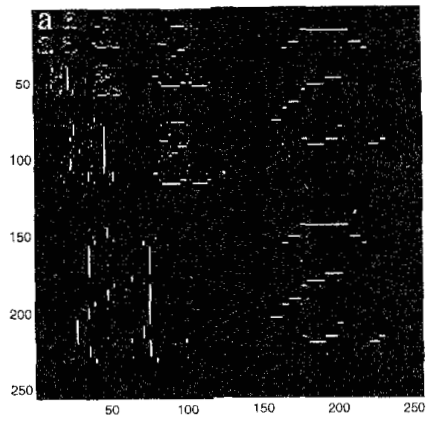

Figure 8: Binary Subband Decomposition of the letter 'a' obtained by the Morphological Subband Decomposition

icant bit plane of the $256 \times 256$ pixels Lena image. This image is created by assigning a 1 to all pixels with values greater than 128 , and 0 to all others. Bit plane images are sequentially transmitted in progressive transmission schemes [4]. The most significant bit plane of the Lena (256x256) image can be compressed 3.01 times whereas a direct LZ coding results in a compression ratio of 2.44 .

This method can be applied to lossless compression of medical images. For $256 \times 256$ pixels segment of an 8-bit mammogram image, the first bit plane was compressed 4.70 times while the LZ compression ratio was 4.00 . For the second bit plane our method's compression was 4.03 whereas $\mathrm{L} Z$ compression ratio was 4.03 . For other bit planes, LZ compression resulted in higher compression ratios. The overall lossless compression ratio is ??.?

\section{CONCLUSION}

In this paper, we describe a nonlinear binary subband decomposition scheme and develop a lossless image compression method based on this decomposition. The compression scheme makes use of a modified form of embedded zero-tree coding. The simulation studies show that the proposed decomposition structure coupled with embedded zerotree coding result in better coding ratios than the Lempel-Ziv coding method.

In this study, we only used the median filter as the building block of the nonlinear subband decomposition structure. Other nonlinear filters can

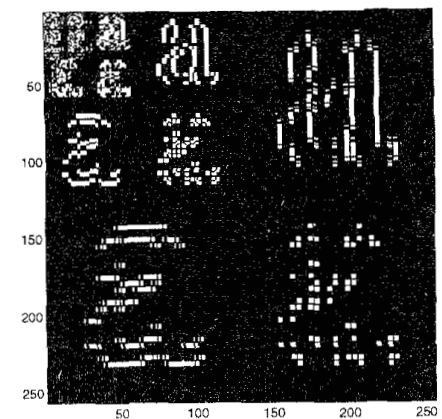

Figure 9: Binary Subband Decomposition of the letter 'a' obtained by The Binary Wavelet Transform

also be used. The effect of the filter on the compression ratio will be investigated in future work.

Another important factor that should be reconsidered is the current bit-compression scheme. Instead of grouping bits and subsequently applying a lossless compression such as run-length coding, a better scheme can be devised. This will also be investigated in the future.

\section{REFERENCES}

[1] O. Egger, M. Kunt, "Embedded Zerotree Based Lossless Image Coding," IEEE ICIP'95, vol. II, pp. 616-619., June 1995.

[2] M. D. Swanson and A. H. Tewfik, "A Binary Wavelet Decomposition of Binary Images," submitted to IEEE Trans. Image Processing.

[3] J. M. Shapiro, "Embedded Image Coding Using Zerotrees of Wavelet Coefficients," IEEE Trans. on Signal Processing, vol. 41, no. 12, pp. 3445 - 3462, Dec. 1993.

[4] M. Rabbani, P. W. Jones, Digital Image Compression Techniques, Tutorial Texts in Optical Engineering, SPIE, 1991.

[5] O. Egger, W. Li, and M. Kunt, "High Compression Image Coding Using and Adaptive Morphological Subband Decomposition," Proceedings of IEEE, vol. 83, no. 2, pp. 272 287, Feb. 1995. 